\title{
ÉCOLE NATIONALE DES CHARTES, Groupe de « La civilisation de l'écrit au Moyen Âge ", Conseils pour l'édition de textes médiévaux
}

\section{Bernard Merdrignac}

\section{(2) OpenEdition \\ 1 Journals}

\section{Édition électronique}

URL: http://journals.openedition.org/abpo/1711

DOI : $10.4000 /$ abpo. 1711

ISBN : 978-2-7535-1483-6

ISSN : 2108-6443

\section{Éditeur}

Presses universitaires de Rennes

\section{Édition imprimée}

Date de publication : 20 septembre 2001

Pagination : 151-153

ISBN : 978-2-86847-625-8

ISSN : 0399-0826

\section{Référence électronique}

Bernard Merdrignac, "ÉCOLE NATIONALE DES CHARTES, Groupe de «La civilisation de l'écrit au Moyen Âge ", Conseils pour l'édition de textes médiévaux ", Annales de Bretagne et des Pays de l'Ouest [En ligne], 108-3 | 2001, mis en ligne le 20 septembre 2003, consulté le 23 septembre 2020. URL : http://journals.openedition.org/abpo/1711; DOI : https://doi.org/10.4000/abpo.1711 
caractère domestique, à l'origine, des fonctions de cet officier responsable du ravitaillement de la cour et chargé de présenter les plats à la table du roi. Mais, par exemple, au XIII ${ }^{\mathrm{e}}$ siècle, Goronwy ab Ednyfed, distain du roi Llywelyn ap Iorwerth, faisait davantage office de Premier ministre que de maître d'hôtel! Aussi l'entreprise de réécriture des cyfreithiau llys par les légistes du Moyen Âge central a eu pour effet de fossiliser les aspects rituels et domestiques des offices de cour. Le parti (délibéré?) de faire l'impasse sur leurs fonctions politiques est sans doute l'expression d'une vision idéale et obsolète d'une royauté festive et répartitive. David Stephenson remarque ainsi qu'alors que les trois principales fêtes du calendrier liturgique (Noël, Pâques et la Pentecôte) sont récurrentes dans ces textes législatifs, pratiquement aucune des chartes qui peuvent être datées durant les XII ${ }^{\mathrm{e}}$-XIII ${ }^{\mathrm{e}}$ siècles n'ont été instrumentées à de telles occasions, censées pourtant renforcer, autour de la cour, la cohésion des couches dominantes de la société.

En bref, l'intérêt de ce recueil collectif dépasse largement le cadre de l'histoire des pays celtiques au Moyen Âge. Les auteurs proposent ici des grilles de lecture appropriées pour aborder certains des témoignages parmi les plus détaillés, mais aussi parmi les plus problématiques, sur la structure et le fonctionnement d'une cour royale dans l'Occident médiéval.

Bernard MERDRIGNAC

ÉCOlE NATIONALE DES CHARTES, Groupe de "La civilisation de l'écrit au Moyen Âge ", Conseils pour l'édition de textes médiévaux. Fascicule I, Conseils généraux, sous la dir. de Françoise Vielliard; fascicule II, Actes et documents d'archives, sous la dir. d'Olivier Guyotjeannin, Paris, CTHS, 2001. 80 F/12,20€ chaque fasc.

Le " retour du texte " sous le sceau duquel s'ouvre le xxI ${ }^{\mathrm{e}}$ siècle pour les médiévistes s'accompagne d'un renouveau de la diplomatique qui n'est d'ailleurs pas toujours forcément perçu à sa juste mesure parmi les spécialistes d'autres périodes historiques. Comme le remarque Emmanuel Poulle, directeur honoraire de l'École des chartes dans l'avant-propos du premier fascicule, les chercheurs français affirment aujourd'hui avec force "la nécessité d'un retour vers l'érudition, avec ses exigences irréductibles en même temps que son intégration délibérée à un questionnement proprement historien ". C'est pourquoi, il faut se féliciter de la diffusion de ces Conseils pour l'édition de textes médiévaux qui constituent la mise en forme définitive des documents de travail internes élaborés à l'École des chartes à l'usage de ses élèves et des membres du groupe de recherche "Civilisation de l'écrit au Moyen Âge " au cours de plusieurs décennies de pratique et de réflexion afin de préciser " les principes qui doivent présider à l'édition des documents médiévaux".

"L'édition est un travail historique à part entière ". Tel est le "Credo " que tout médiéviste est prêt à conjuguer avec les éditeurs de ces fascicules. La réflexion théorique en matière d'édition critique des textes littéraires, dans la mesure où elle a davantage prêté à controverses, est plus avancée que celle qui concerne les " sources documentaires " dont les éditeurs doivent faire avec le pragmatisme qui a longtemps prévalu chez leurs prédécesseurs. Le parti de traiter l'ensemble des disciplines concernées par l'art de l'édition constitue donc de la part des coordonnateurs de ce travail d'équipe une démarche originale qui rend compte de l'architecture de la présente publication. " On a d'a- 
bord cherché à regrouper, toutes catégories de textes confondues, des conseils généraux dont tout médiéviste puisse faire son profit (fasc. 1) avant d'aborder les problèmes spécifiques aux actes et documents d'archives (fasc. 2), puis aux textes littéraires " qui seront traités dans un troisième fascicule dont la parution est annoncée sous peu. À juste titre, les auteurs se montrent réticents à manier l'anathème et préconisent d'adapter les règles au document (rareté, ancienneté relative, état de conservation) et au destinataire visé (édition " courante ", " de recherche ", à finalité " paléographique ou pédagogique "...). Il n'en demeure pas moins que les conseils prodigués ici n'expriment pas seulement leurs positions personnelles, mais qu'ils " constituent le socle même de la doctrine chartiste en matière d'édition " (E. Poulle).

C'est pourquoi, comme le revendiquent les éditeurs avec insistance (fasc. 1, p. 63, n. 1), ces règles d'édition reflètent la pratique française, « les autres pratiques européennes étant notablement différentes ». Dans les limites de ce compte-rendu, il suffit simplement de rappeler, par exemple, qu'en matière de ponctuation, il est convenu de rétablir l'usage moderne (quitte, le cas échéant, à étudier à par le système de ponctuation employé par le scripteur) ou qu'en ce qui concerne les majuscules, il est recommandé ne pas tenir compte de la pratique du scribe, mais de se rapprocher autant que faire se peut de l'usage contemporain. De même, à la différence de leurs collègues britanniques, entre autres, les éditeurs français de textes en latin médiéval s'attachent, en règle générale, à distinguer " $\mathrm{u}$ " de " $\mathrm{v}$ ", ou " $\mathrm{i}$ " et " $\mathrm{j}$ " (interjecta et non interiecta). Il est à souhaiter qu'en viennent à s'atténuer ces divergences héritées de traditions différentes en matière d'érudition et de méthode historique. En effet, pour formelles qu'elles soient, ces discordances risquent d'être source de malentendus dans le cas d'un texte se rattachant à une aire culturelle donnée de la chrétienté médiévale et édité selon les principes spécifiques à une école historique nationale de l'Europe contemporaine.

Pour illustrer cette préoccupation, il n'est que de se reporter aux remarques que formulait, en 1991, Neil Wright dans son édition critique des Gesta regum Britannie ${ }^{1}$, à l'encontre de Francisque Michel qui en avait proposé une édition semi-diplomatique en 1862 à l'intention de la Cambrian Archaelogical Association ${ }^{2}$ ! Ce poème composé en Bretagne continentale au milieu du XIII ${ }^{\mathrm{e}}$ siècle et dédié à l'évêque Cadioc de Vannes (1236-1254) est une réécriture de l'Historia Regum Britannie de Geoffroy de Monmouth sur le modèle de l'Alexandreis de Gauthier de Chatillon. Contentons-nous de juxtaposer quelques vers, retenus presque au hasard, des deux éditions successives :

Ecce viris plene, vente rapiente, carine

Octodecim veniunt, quas jam ${ }^{3}$ Germania flava

Miserat Engisto; cujus quoque filia Rowen ${ }^{4}$

Virgo venit, facie regni prelata puella. (F. Michel, l. V, v. 2221-2224.)

1. WriGHT, N., ed. and translat., The Historia Regum Britannie of Geoffrey of Monmouth - V. Gesta Regum Britannie, Cambridge, D. S. Brewer, 1991 - cf. le compte rendu dans les $A B P O$, t. $100, \mathrm{n}^{\circ} 2$ (1993), p. 235-237.

2. Michel, F., (ed.), Gesta Regum Britannie, A metrical history of the Britons of the XIIIth century new first printed from three manuscripts, printed for the Cambrian Arcaelogical Association, Bordeaux, 1862.

3. Var. : illas.

4. Var. : Ronven. 
Ecce uiris plene uento rapiente carine

Octodecim ueniunt quas iam ${ }^{5}$ Germania flaua

Miserat Hengisto ${ }^{6}$. Cuius quoque filia Ronuen

Uirgo uenit, facie regni prelata puellis. (N. Wright, l. V, [100] v. 271-275.)

L'art d'éditer a donc une histoire, et chacun des deux fascicules est enrichi de nombreuses études de cas, fac simile, et documents commentés qui viennent étayer le propos des auteurs. Il a aussi une actualité : comme l'annoncent ici Olivier Guyotjeannin et Françoise Vielliard, il est à prévoir que " La juxtaposition d'entreprises pour l'heure assez éclatées en matière de mise à disposition des textes médiévaux sur supports électroniques (éditions sur Internet, numérisation de manuscrits et d'actes, saisie de textes interrogeables à distance sur CD Rom) amènera sans aucun doute à repenser les stratégies d'édition ". Bref, dans l'attente du troisième fascicule consacré aux " textes littéraires ", voici un travail collectif qui rendra de grands services à la " communauté des maîtres et des étudiants " en histoire du Moyen Âge. On ne saurait trop en recommander la pratique à ces derniers dès l'année de la maîtrise, voire même auparavant. Est-il nécessaire de rappeler ici que le superbe album de documents commentés autour de Gerbert d'Aurillac, publié naguère sous la direction d'Olivier Guyotjeannin et d'Emmanuel Poulle ${ }^{7}$ est un modèle d'édition de textes médiévaux et constitue un pourvoyeur inépuisable de sujets de Travaux Dirigés.

Bernard MERDRIGNAC

Le Livre du Graal, t. I, Joseph d'Arimathie - Merlin - Les Premiers faits du roi Arthur, édition préparée par Daniel Poirion, publiée sous la direction de Philippe Walter, avec, pour ce volume, la collaboration d'Anne Berthelot, Robert Deschaux, Irène Freire-Nunes et Gérard Gros, Gallimard, NRF, Bibliothèque de la Pléiade, Paris, 2001, 2000 p., 450 F/68,83 €.

À la suite du décès de Daniel Poirion, Philippe Walter, professeur de littérature française du Moyen Âge à l'Université de Grenoble III, a pris la direction de l'édition préparée par La Plë̈ade du cycle romanesque majeur du XIII siècle que les médiévistes intitulent " cycle de la Vulgate ", depuis l'édition de H. Oskar Sommer en 1909, et qui est connu du grand public, généralement par le biais d'adaptations plus ou moins fidèles, sous le nom de "romans de la Table Ronde ". On appelle habituellement Lancelot-Graal, le roman de Lancelot proprement dit, suivi de La Quête du Saint Graal et de La mort du roi Arthur. Ces trois récits constitueront la matière des tomes II et III, à paraître ultérieurement. Les éditeurs du présent volume présentent ici les trois volets préliminaires de cette somme romanesque que sont Joseph d'Arimathie, récit des " enfances " du Graal, Merlin, magicien et prophète, qui fonde la Table ronde et les Premiers faits du roi Arthur qui campent la stature mythique de ce souverain exemplaire. Les éditeurs ont retenu pour titre d'ensemble de ces six textes

5. Var. : illas.

6. Var. : Engisto.

7. Guyotjeannin, O., Poulle, E., Autour de Gerbert d'Aurillac, le pape de l'an Mil, Matériaux pour l'histoire publiés par l'École des chartes, Paris, École des Chartes, 1996. 